\title{
Autism and Oxytocin: New Developments in Translational Approaches to Therapeutics
}

\author{
Joshua J. Green and Eric Hollander \\ Montefiore Medical Center, University Hospital of Albert Einstein College of Medicine, Bronx, New York 10467
}

\begin{abstract}
Summary: Autism is a neurodevelopmental disorder characterized by dysfunction in three core symptom domains: speech and communication deficits, repetitive or compulsive behaviors with restricted interests, and social impairment. The neuropeptide oxytocin, along with the structurally similar peptide arginine vasopressin, may play a role in the etiology of autism, and especially in the social impairment domain. Oxytocin is a nonapeptide (i.e., it has nine amino acids). It is synthesized in magnocellular neurons in the paraventricular nucleus and the supraoptic nucleus of the hypothalamus and is released into the bloodstream by way of axon terminals in the posterior pituitary. Oxytocin is released both peripherally, where it is involved in
\end{abstract}

milk letdown and the facilitation of uterine contractions, and centrally, where it acts as a neuromodulator along with arginine vasopressin. Here, we discuss relevant translational research pertaining to the role of oxytocin in social and repetitive behaviors and consider clinical implications. We also discuss current research limitations, review recent preliminary findings from studies involving oxytocin in autism spectrum disorder patient populations, and point to possible directions for future research. Key Words: Autism, ASD, oxytocin, translational model, repetitive behavior, social behavior, social cognition, social functioning, therapeutics, arginine vasopressin, epigenetic.

\section{INTRODUCTION}

Autism is a neurodevelopmental disorder characterized by dysfunction in three core symptom domains: speech and communication deficits, repetitive or compulsive behaviors with restricted interests, and social impairment. ${ }^{1}$ The social impairment symptom domain involves deficits typified by a lack of eye contact, lack of social and emotional reciprocity, lack of mutual enjoyment in social activities, diminished ability to hold a conversation, and impaired daily interaction skills. ${ }^{2}$ Interaction partners are often left with an impression of a socially detached, unreceptive, or somewhat odd person. $^{2}$ There also appear to be deficits in social recognition $^{3-5}$ and in recognition of facial expressions, age, and sex, ${ }^{6,7}$ as well as in face processing. The repetitive behaviors domain involves restrictive interests, rigid routines or ritualistic behaviors, stereotypies, self-stimulatory behaviors, and a preoccupation with parts of objects. $^{2}$

Address correspondence and reprint requests to: Eric Hollander, M.D., Director, Compulsive, Impulsive and Autism Spectrum Disorders Program, Child Psychiatry Annex, 111 E. 210th Street, Bronx, NY 10467. E-mail: ehollander@montefiore.org.
During recent decades, several researchers have suggested that the neuropeptide oxytocin (OXT) may play a role in the etiology of autism. ${ }^{2,8-16}$ Oxytocin is a nonapeptide (i.e., it has nine amino acids) that is synthesized in magnocellular neurons in the paraventricular nucleus and the supraoptic nucleus of the hypothalamus and is released into the bloodstream by way of axon terminals in the posterior pituitary. ${ }^{17,18}$ It is released both peripherally, where it is involved in milk letdown and the facilitation of uterine contractions, and centrally, where it acts as a neuromodulator along with the structurally similar peptide arginine vasopressin (AVP). ${ }^{18}$

Of specific interest here, the neuromodulatory role of OXT (and AVP) is critically involved in affiliative and sexual behaviors, including formation of mother-infant and adult-adult pair bonds, separation distress, and social memory and recognition, as well as stress response and regulation of feeding and grooming. ${ }^{14}$ Because social impairment and sociocognitive deficits are core features of autism spectrum disorders (ASD), we and oth$\mathrm{ers}^{2,8-16}$ have proposed that OXT may be a factor in the pathophysiology of ASD. Even though there is no primary deficit of the OXT system in most patients with ASD, the OXT system can nevertheless be manipulated at various stages in development, which can result in the 
rescue of certain core symptom domains that characterize ASD. Although significant research has been done on the role of AVP as well, the chief focus here is on OXT.

In this review, we discuss translational research implicating OXT in affiliative and social behaviors and in repetitive-compulsive or stereotyped behaviors. We also briefly address the current advantages and limitations of animal models. Next, we review the most recent preliminary studies suggesting the efficacy of exogenous OXT in subjects with ASD, as well as recent genetic and epigenetic research findings. Finally, we conclude with suggestions for future research and discuss current limitations and challenges in studying the links between OXT and autism.

\section{TRANSLATIONAL RESEARCH}

\section{Oxytocin and social behaviors in rats and mice}

A substantial body of animal research over the past three decades suggests that OXT (and also AVP) plays a critical role in social aspects of behavior, such as social recognition via olfaction, ${ }^{19}$ social memory, ${ }^{20}$ pair bonding (including the mother-infant bond), ${ }^{21,22}$ and maternal behavior. ${ }^{23}$ Rat studies of maternal behavior show that, despite the finding that virgin female rats generally avoid pups and find them aversive,${ }^{24}$ maternal behavior can be induced via central administration of OXT. ${ }^{25}$ Conversely, the onset (but not maintenance) of maternal behavior can be inhibited with central administration of OXT antagonists. ${ }^{26}$ Moreover, Champagne et al. ${ }^{27}$ found that female rats who showed increased maternal behavior with pups, as defined by increased licking and grooming, also showed significantly higher oxytocin receptor levels in the medial preoptic area, the lateral septum, the central nucleus of the amygdala, the paraventricular nucleus of the hypothalamus, and the bed nucleus of the stria terminalis.

Additional evidence has been found in studies of genetically modified mice. For example, Takayanagi et al. ${ }^{28}$ genetically modified mice for a null mutation in the oxytocin receptor gene (Oxtr) and compared them with OXT-deficient mice. The myometria of Oxtr-null dams [maternal mice who were oxytocin receptor negative (Oxtr-I-)] failed to contract in response to either endogenous oxytocin or arginine vasopressin (AVP), a partial agonist of the oxytocin receptor. In addition, these mice had decreased maternal nurturing and decreased lactation, thus conferring poorer survival in their offspring. Oxtr-null infant males vocalized less when in social isolation than their normal control counterparts, ${ }^{28}$ confirming findings from previous studies. ${ }^{29-30}$ This suggests that animals lacking the ability to respond properly to oxytocin are significantly less distressed by social isolation or by the absence of social attachment/stimuli. However, the OXT-deficient mice (normal receptor expres- sion but lacking oxytocin hormone) exhibited normal maternal behavior, ${ }^{28}$ suggesting that other ligands such as AVP can activate the Oxtr protein product and thus compensate for the OXT deficiency. This is in accord with findings from other studies indicating that OXT knockout mice show normal mating, parturition, and maternal behavior. ${ }^{29,30}$

Both OXT and AVP also play a role in social cognition in rodents via the olfactory system. Social recognition may be measured in rodents by the duration of olfactory investigation of other rodents. Although OXT knockout mice fail to recognize a novel mouse after repeated exposures, ${ }^{20,31}$ this deficit can be rescued with a single intracerebroventricular injection of OXT just before the initial encounter. ${ }^{32}$ Such a finding implies that OXT is crucial for the formation of social memory and is involved in social recognition in rodents.

\section{Oxytocin and social behaviors in prairie voles}

The prairie vole (Microtus ochrogaster) is another rodent species especially relevant to studies involving social affiliation and pair bonding. Prairie voles are known to show remarkable individuality in the expression of social behavior, including partner preference after mating, a trait not generally found in other rodents. ${ }^{33}$ They also exhibit spontaneous maternal nurturing, a behavior found in roughly half of all virgin female prairie voles. ${ }^{34}$ Perhaps most intriguing, field studies have revealed that male prairie voles are generally monogamous, and that once a pair bond is established they generally form longterm partnerships, sharing a nest and home throughout the breeding season and tending to travel together. ${ }^{35-37}$ Additionally, the pair remain together until death and when one dies the survivor does not usually form a new pair bond. ${ }^{38,39}$ Prairie voles thus provide an excellent animal model for social behavior, and a sizeable body of research attests to this fact. ${ }^{33-42}$

Considerable evidence for the role of OXT in these social and affiliative behaviors has been accumulated since the early 1980s. In terms of partner preference, female prairie voles usually need to mate in order to establish a partner preference; however, it has been found, first, that a central administration of OXT can induce partner preference without mating ${ }^{41,42}$ and, second, that the formation of these partner preferences can be mitigated by administering an OXT antagonist just prior to mating. ${ }^{42}$ Additionally, virgin female prairie voles that demonstrated maternal nurturing behavior had significantly higher densities of OXT receptor in the nucleus accumbens than did those that displayed no maternal behaviors. ${ }^{43}$ Again, such display of alloparental behavior can be extinguished with the infusion of an OXT antagonist into the nucleus accumbens. ${ }^{43}$ These findings imply that OXT is involved not only in reproductive functions, but also in individual differences in 
maternal behavior, as well as in partner preference and pair bonding.

\section{Oxytocin and repetitive behaviors}

Oxytocin has a decidedly lesser role in the expression of repetitive behaviors, but it nonetheless has been implicated in various repetitive behaviors in animal models. Indeed, intracerebroventricular administration of OXT has been found to induce stereotyped behaviors such as stretching, repetitive grooming, and squeaking in mice, ${ }^{44-48}$ grooming in rats, ${ }^{45}$ and wing-flapping in chicks. ${ }^{15}$ Although little translational research has been conducted to investigate the relationship between OXT and repetitive behaviors, other research highlights the apparent link between OXT and obsessive-compulsive disorder $(\mathrm{OCD})$, a condition characterized by repetitive behaviors and unwanted obsessional thoughts. Additionally, the dopamine and serotonin systems of the brain are implicated in OCD, and OXT is known to interact with these systems. ${ }^{49}$ Moreover, it has been found that pregnancy and the puerperium (both being periods when OXT levels fluctuate) are associated with OCD onset or a worsening of symptoms. ${ }^{49}$ These findings offer support for the suggestion that OXT is related to the manifestation of repetitive behaviors, although more research is needed to clarify its role.

\section{Advantages and limitations of animal models}

In the study of autism, the chief limitation of animal models is that humans develop autism but animals do not. Thus, animal research can address only various manifestations of the symptoms associated with ASD. This limits the types of generalizations and translations that can be made from animal to human models.

There are now several animal models of autism (see, for example, Crawley's 2004 article, ${ }^{50}$ and a brief review from 2006 by Ashwood et al. ${ }^{51}$ ) that are useful for studying various aspects of autism that could not be tested directly in humans because of ethical limitations. The main benefits of working with these animal models are that 1) oxytocin receptors and peptide levels can be directly measured in brain tissue, 2) agonists and antagonists of OXT can be administered centrally, and 3) various ligands can be used to measure central OXT receptor binding.

\section{CLINICAL IMPLICATIONS}

The fruits of 30 years of animal research offer compelling evidence that any solution of the complex puzzle of ASD will call for a thorough understanding of the neurobiological roles of OXT (and AVP) in social and repetitive behaviors. We are still at the beginnings of reaching such understanding, and thus cannot concretely define the exact roles of OXT and AVP in human social and repetitive behavior. Nevertheless, preliminary research shows promising results.

\section{Oxytocin in healthy control populations}

In a now highly cited and distinctive study, Kosfeld et al. ${ }^{52}$ found that intranasal OXT increases trust in healthy male control subjects. Specifically, volunteers played the trust game, in which participants are given money that they may invest (some of it or all of it) with an anonymous trustee. The trustee promises to triple the amount of money invested, but there is always the risk that the trustee will break this trust bond in order to make higher profits. Those participants who received intranasal OXT invested the most money, which can be interpreted as reflecting increased trusting behavior (the investigators had controlled for the alternative explanation, that OXT might have simply increased risky behavior in participants). It is worth emphasizing here that OXT appears to increase trust, even in the face of a negative first impression; that is, when the trustee breaks the trust, control subjects typically decrease the amount of money they are willing to invest on the next transaction, whereas the OXT group continued to trust in what would normally be seen as an untrustworthy partner. ${ }^{53}$ In this sense, it appears that OXT may increase the emotional and behavioral components of trust, while thwarting the cognitive processing component. A cognitive deficit of this sort is not uncommon in ASD.

A second study on OXT and healthy control subjects, one more relevant to autism, assessed the effect of OXT on performance on the Reading the Mind in the Eyes Test (RMET) ${ }^{54}$ In this test, the subject matches photographs showing only the eye region with possible mental or emotional descriptors. It is generally accepted that this test measures the ability to infer the mental state of another person. Because it has been shown that patients with ASD generally do poorly on this task, ${ }^{54}$ it is especially exciting to learn that healthy non-ASD control subjects perform better on this test with OXT than with placebo. ${ }^{55}$ Finally, it has been shown that OXT also increases the perceived trustworthiness and attractiveness of the human face, ${ }^{56}$ and that OXT may enhance the buffering effect of social support in humans, ${ }^{8}$ because it has been shown to decrease the stress response during a socially stressful situation. ${ }^{57}$

\section{Oxytocin in ASD patient populations: biological and therapeutic research findings}

Oxytocin blood plasma levels. Some of the first studies to investigate the relation of OXT to ASD compared OXT blood plasma levels between control subjects and patients with ASD. For example, Modahl et al. ${ }^{58}$ measured the midday plasma samples of OXT in prepubescent autistic and age-matched normal children and found that, even after controlling for individual variation, the autistic children had significantly lower plasma OXT 
levels. Additionally, OXT levels were positively correlated with age in normal control subjects, but not in the autistic children; however, conclusions from this study may be drawn only with reservations, because of the peripheral vs. central limitation discussed under Current Challenges and Limitations. Moreover, lower scores on social and developmental measures were associated with the autistic children and were lowest in a subset of the autistic children who were characterized as 'aloof. ${ }^{58}$ Another study, however, found the opposite results: in an adult population, ASD patients had higher OXT plasma levels than control subjects. ${ }^{59}$ To account for this, it has been suggested that OXT is processed differently in the brains of ASD patients, rather than simply at a different level. ${ }^{60}$

Overall, the relationship between OCT and ASD remains unclear. It has been speculated that the seemingly contradictory findings might have to do with the fact that the two studies involved different populations (adults ${ }^{59}$ vs. children ${ }^{58}$ ) and that the study with the adults involved a less homogeneous sample with respect to age and level of functioning. Additionally, Bartz and Young ${ }^{61}$ note that other research suggests a relationship between elevated OXT plasma levels and self-reports of relational distress or dissatisfaction with relationships, ${ }^{62,63}$ as well as with increased social anxiety, ${ }^{64}$ a trait not uncommon in many adults with ASD.

Functional MRI research. More recently, genetic research and findings from neuroscience have offered some intriguing insights into the possible etiology of ASD and related disorders. First, research using functional magnetic resonance imaging (fMRI) has indicated that intranasal OXT tapers down activity in brain regions associated with mediating fear and arousal, such as the amygdala and the midbrain, when exposed to fear-inducing stimuli. ${ }^{65}$ Moreover, similar to the effect on trust, Domes et al. ${ }^{66}$ found that OXT appears to reduce activity in the amygdala, regardless of whether participants are shown happy, angry, or fearful faces. Again, this suggests that OXT affects only certain percepts or components of various social deficits, and that the neurobiological mechanisms are still only moderately understood. Nonetheless, the literature supports the notion that OXT is involved in the mediation of the fear response and of the level of arousal to certain social and emotional stimuli. It could be that OXT facilitates certain types of social behavior via a reduction of arousal to social and social anxiety-causing stimuli. ${ }^{61}$ Indeed, other studies confirm that OXT is associated with a reduction in amygdala activity. ${ }^{67,68}$

Paradoxically, in the most recent study by Domes et al. ${ }^{69}$ the opposite effect was found. Participants were administered intranasal OXT or placebo and shown fearful, angry, happy, or neutral facial expressions while fMRI was performed. When subjects viewed fearful faces, the blood oxygen level dependent (BOLD) signal was enhanced, rather than decreased, in the left amygdala, the fusiform gyrus, and the superior temporal gyrus. When subjects viewed happy or angry faces, activity was again increased, but only in the inferior frontal gyrus. The results were independent of OXT plasma levels. These findings highlight the need, as the authors suggest, for similar research involving both sexes (the Domes et al. ${ }^{69}$ study involved only women, and previous studies involved only men). Given this new evidence, the relationship between amygdala activity and OXT now appears to be more complex than previously thought, although Domes et al. ${ }^{69}$ speculate a possible sexual dimorphism in the neutral effects of OXT and a role of gonadal steroids and OXT receptor affinity.

Genetic and epigenetic research. Given that ASD appears to have both the highest monozygotic twin concordance rate $(73-95 \%)^{70}$ and the highest heritability estimate (estimated at $>90 \%$ ), ${ }^{70-72}$ it is widely accepted that autism is a highly heritable neurodevelopmental disorder. Not surprisingly, then, much research on the etiology of autism has sought to find genetic factors. Because autism appears to have a male:female sex ratio of $4: 1$, the involvement of the $\mathrm{X}$ chromosome has received considerable attention. ${ }^{70}$ In addition, neuroimaging and neuroanatomical studies support a prenatal origin of ASD, with neurodevelopmental abnormalities identified in the first two trimesters of pregnancy. ${ }^{70}$

Because of technological constraints, nearly all of the research concerning the genetics of autism has been done in the 21 st century, and we are only at the beginning of the pursuit of full comprehension of the genetic factors involved in ASD.

As already noted for animal research, much of the human genetics research has focused on the OXTR gene, and several studies have provided evidence for $O X T R$ in the pathophysiology of $\mathrm{ASD}^{73-76}$ (and also one or more of the AVP receptor genes). One initial study, on a group of 195 Han Chinese parent-offspring autism trios, found a significant association between two single nucleotide polymorphisms (SNPs) in OXTR (rs2254298 and rs53576) and ASD. ${ }^{73}$ A study designed to replicate these findings in a European-origin population found similar results: a significant association was detected at rs2254298 $(p=0.03)$ but not rs53576. ${ }^{74}$ Moreover, for the rs2254298 they recorded an overtransmission of the $\mathrm{G}$ allele to probands with autistic disorder, whereas overtransmission of the A allele was found in the Chinese Han population; in both cases, however, the $\mathrm{G}$ allele was more frequent than the A allele. ${ }^{73,74}$

Two additional studies offer further support for the role of OXTR in ASD. ${ }^{75,76}$ Lerer et al..$^{75}$ found an association between ASD and the SNP rs2254298, but not rs53576. Additionally, they found that the rs2254298 SNP was also associated significantly with lower scores 
in the daily living skills and communication domains of the Vineland Adaptive Behavior scale. The second study also found associations with a single SNP and a haplotype; the authors concluded that genetic variation in the OXTR gene may be relevant to the etiology of autism on a high-functioning level. ${ }^{76}$

Until recently, research into the genetic etiology of autism yielded little in conclusive findings, but a recent study suggests involvement of not only genetic but also epigenetic factors. In an investigation of a variety of genomic and epigenetic evidence for OXTR deficiency in autism, Gregory et al. ${ }^{77}$ identified a deletion in the OXTR gene in an autism proband and in his mother with OCD; moreover, an affected sibling had epigenetic misregulation of this gene through aberrant gene silencing by DNA methylation. Increases in DNA methylation in the $\mathrm{CpG}$ island known to regulate OXTR expression were found in peripheral blood cells and temporal cortex in independent datasets of individuals with autism, compared with control subjects. Increased methylation was associated with decreased OXTR mRNA expression in temporal cortex. These data implicate epigenetic regulation of OXTR in the development of autism.

Therapeutic research for the repetitive-compulsive behavior domain. To date, there has been only minimal research to investigate the role of OXT with respect to repetitive behaviors seen in ASD. In one study, Hollander et al. ${ }^{10}$ conducted a double-blind, placebo controlled, cross-over study in which synthetic OXT (Pitocin) was administered to 15 adults with ASD via intravenous infusion in a randomized, counter-balanced design in which subjects served as their own controls. Participants underwent two identical challenge days in which they were administered a continuous infusion of OXT or placebo intravenously over a 4-hour period and were evaluated for severity and frequency of repetitive behaviors using a four-point ordinal scale ranging from 0 (never) to 3 (constantly). The ratings were completed at baseline ( 0 minutes) and at $60,120,180$, and $240 \mathrm{~min}$ utes. The results of a repeated-measures analysis of variance indicated that the severity and frequency of repetitive behaviors, as well as the total number of different repetitive behaviors, decreased over time after OXT administration, compared with placebo. ${ }^{10}$

These results suggest that OXT may play a role in severity and frequency of common compulsive and repetitive behaviors seen in ASD and offer further support for the theory that OXT dysfunction plays a role in the etiology of autism. Nonetheless, the Hollander et al. ${ }^{10}$ study raises some interesting questions. As already noted, animal research has shown that OXT administration can induce or increase stereotyped behaviors, ${ }^{15,44-48}$ which raises the question of why one would expect OXT to reduce rather than increase repetitive behaviors in humans with ASD. In humans, studies in OCD associate greater OXT levels with greater frequency of repetitive behaviors, ${ }^{2,10}$ which contrasts with studies in ASD that suggest the opposite, revealing important differences between these disorders. As Bartz and Hollander ${ }^{2}$ suggest, it may be that too much OXT or an increased sensitivity to OXT is implicated in OCD, whereas a deficit of OXT is implicated in ASD. Overall, it seems plausible that OXT has different effects in the treatment of the repetitive or compulsive behaviors in ASD and in OCD.

Therapeutic research for the social impairment domain. Support for the role of OXT in the social impairment seen in ASD has grown considerably in the last decade. As already noted, patients with ASD generally perform poorly on the Reading the Mind in the Eyes Test (RMET),${ }^{54}$ because of the sociocognitive deficits associated with understanding the internal states of others (referred to as theory of mind).$^{54,61}$ They also display deficits in affective speech comprehension ${ }^{78,79}$ and in identifying the affective states of others as measured by emotions portrayed through facial expressions. ${ }^{7,78} \mathrm{Be}-$ cause it has been shown that healthy control subjects improve on the RMET with OXT, ${ }^{54}$ similar studies have been conducted with ASD populations.

Using the same population and a similar method as in the study described above for repetitive behaviors, Hollander et al. ${ }^{9}$ also measured the comprehension of affective speech in individuals with ASD. Specifically ${ }^{\circ}$, they tested the ability to assign an emotional significance to certain intonations in speech. Mixed regression analysis indicated that there was indeed a significant improvement on the affective comprehension task, and that those individuals who had received OXT infusion on the first visit showed retention of the ability on the second visit, 2 weeks later. ${ }^{9}$ This finding implies that oxytocin administration enhances the development of social memories. More recently, additional support has been found in an adolescent population. ${ }^{80}$ Specifically, in a study with a population age range of 12-19 years, participants with ASD improved significantly on the RMET with an intranasal delivery method; furthermore, when the analysis was restricted to the younger participants aged 12 to 15 , who received a lower dose, the findings were still significant. ${ }^{80}$

Eye gazing has also been studied in relation to the social impairment seen in ASD. Patients with ASD spend less time gazing at the eyes when looking at images of people than do normal control subjects. One study found that ASD patients not only gazed less at the eyes but also gazed more at the mouth region, compared with control subjects. ${ }^{81}$ In support of the role of OXT in this behavior, it has been found OXT does increase gazing to the eye region, compared with placebo. ${ }^{82}$

Both OXT and AVP have been shown to facilitate social memory in rodents. On that basis, a study investigating OXT and social memory would be useful. Al- 
though to date this has not been investigated in an ASD population, Rimmele et al. ${ }^{83}$ found that OXT improved memory for faces in healthy control subjects, and, importantly, did not improve memory for other types of nonsocial stimuli. Overall, preliminary research appears to suggest a significant role of OXT in social memory and in some of the social deficits that characterize ASD.

\section{CURRENT CHALLENGES AND LIMITATIONS}

Several important challenges and limitations are associated with the body of research on ASD. First, patients with ASD vary widely in their manifestation of symptoms and in the severity of their social impairments, language deficits, and repetitive-compulsive behaviors. The effect of such heterogeneous samples must not be overlooked in research design and interpretation. Parallel to this, the complete etiology of autism and ASD is not fully understood, and there appear to be multiple causes. Indeed, fewer than $10 \%$ of autism cases are attributed to single-gene disorders. ${ }^{77}$ Furthermore, in the presence of heterogeneity, multiple contributing loci, and different gene-environment interactions, genetic factors linked to autism are highly complex. ${ }^{84}$

In human studies, and in contrast to animal research, it is difficult to administer a drug or take measurements centrally, because of the invasive nature of such procedures. With the exception of the recent epigenetic research by Gregory et al. ${ }^{77}$ this significantly limits research. Because of the blood-brain barrier, OXT levels in the blood do not generally signify corresponding levels in the brain, and it is unknown what amount of OXT reaches the brain when it is administered peripherally. In considering the epigenomics of both peripheral blood cells and cells in the temporal cortex, Gregory et al. ${ }^{77}$ found DNA methylation binding on the OXTR gene in peripheral measures to be tightly correlated with brain epigenomic measures. Animal models of social behavior are generally based on olfaction projections to subcortical areas modulated by OXT (and AVP). In humans, social cognition involves several additional areas of the brain, including visual, auditory, attentional, and executive control regions. ${ }^{8}$

In terms of effect of OXT on trust, it is important to note that trusting difficulties are not a characteristic of ASD, especially because patients with ASD are sometimes so sociocognitively removed from social discourse that they do not fully understand what it means to trust someone. Other limitations in human research include the need for sensitive, valid, and reliable outcome measures of the social cognition domain, the lack of PET ligands for the OXT receptor in humans, and the lack of available small molecule agonists for use in humans.

\section{FUTURE DIRECTIONS}

In sum, the last two decades have seen an increasing accumulation of translational research aimed at better understanding the neurobiological relationship of OXT (and AVP) to social behavior. Additionally, the most recent decade has seen compelling preliminary research in ASD patient populations. Further work is needed to fully determine the safety and efficacy of OXT and potential OXT receptor agonists in adults and children with ASD. Additional work is also needed to clarify which symptom domains are most reliably influenced, which patients may be most responsive, and by what mechanisms OXT exerts its behavioral effects.

Acknowledgments: The authors acknowledge funding by the National Alliance for Research on Schizophrenia and Depression (NARSAD), the Seaver Foundation, and the National Institute of Mental Health (NIH-NIMH) Studies to Advance Autism Research and Treatment (STAART) centers. E.H. is named as an inventor on a patent application for oxytocin in autism.

\section{REFERENCES}

1. American Psychiatric Association; Task Force on DSM-IV. Diagnostic and statistical manual of mental disorders (DSM-IV). 4th ed. Washington, DC: American Psychiatric Association, 1994.

2. Bartz J, Hollander E. Oxytocin and experimental therapeutics in autism spectrum disorders. Prog Brain Res 2008;170:451-462.

3. Szatmari P, Tuff L, Finlayson MA, Bartolucci G. Asperger's syndrome and autism: neurocognitive aspects. J Am Acad Child Adolesc Psychiatry 1990;1:130-136.

4. Davies S, Bishop D, Manstead AS, Tantam D. Face perception in children with autism and Asperger's syndrome. J Child Psychol Psychiatry 1994;6:1033-1057.

5. Barton JJ. Disorders of face perception and recognition. Neurol Clin 2003;2:521-548.

6. Weeks SJ, Hobson RP. The salience of facial expression for autistic children. J Child Psychol Psychiatry 1987;28:137-151.

7. Tantam D, Monaghan L, Nicholson H, Stirling. Autistic children's ability to interpret faces: a research note. J Child Psychol Psychiatry 1989;30:623-630.

8. Hammock EA, Young LJ. Oxytocin, vasopressin and pair bonding: implications for autism. Philos Trans R Soc Lond B Biol Sci 2006;361:2187-2198.

9. Hollander E, Bartz J, Chaplin W, et al. Oxytocin increases retention in social cognition in autism. Biol Psychiatry 2007;61:498 450 .

10. Hollander E, Novotny S, Hanratty M, et al. Oxytocin infusion reduces repetitive behaviors in adults with autistic and Asperger's disorders. Neuropsychopharmacology 2003;28:193-198.

11. Insel TR. A neurobiological basis of social attachment. Am J Psychiatry 1997;154:726-735.

12. Insel TR, O'Brien DJ, Leckman JF. Oxytocin, vasopressin and autism: Is there a connection? Biol Psychiatry 1999;45:145-157.

13. Lim MM, Bielsky IF, Young LJ. Neuropeptides and the social brain: potential rodent models of autism. Int J Dev Neurosci 2005; 23:235-243.

14. McCarthy MM, Altemus M. Central nervous system actions of oxytocin and modulation of behavior in humans. Mol Med Today 1997;3:269-275.

15. Panksepp J. Oxytocin effects on emotional processes: separation distress, social bonding, and relationships to psychiatric disorders. Ann N Y Acad Sci 1992;652:243-252.

16. Waterhouse L, Fein D, Modahl C. Neurofunctional mechanisms in autism. Psychol Rev 1996;103:457-489. 
17. Insel TR, Young LJ. Neuropeptides and the evolution of social behavior. Curr Opin Neurobiol 2000;10:784-789.

18. Burbach JPH, Young LJ, Russell JA. Oxytocin: synthesis, secretion, and reproductive functions. In: Neill JD, editor. Knobil and Neill's physiology of reproduction. 3rd ed. Amsterdam: Elsevier, 2006:3055-3128

19. Kendrick KM, Da Costa APC, Broad KD, et al. Neural control of maternal behaviour and olfactory recognition of offspring. Brain Res Bull 1997;44:383-395.

20. Ferguson JN, Young LJ, Hearn EF, Matzuk MM, Insel TR, Winslow JT. Social amnesia in mice lacking the oxytocin gene. Nat Genet 2000;25:284-288.

21. Donaldson ZR, Young LJ. Oxytocin, vasopressin, and the neurogenetics of sociality. Science 2008;322:900-904.

22. Young LJ, Wang Z. The neurobiology of pair bonding. Nat Neurosci 2004;7:1048-1054.

23. Perdersen CA, Prange AJ Jr. Induction of maternal behavior in virgin rats after intracerebroventricular administration of oxytocin. Proc Natl Acad Sci U S A 1979;76:6661-6665.

24. Fleming AS, Anderson V. Affect and nurturance: mechanisms mediating maternal behavior in two female mammals. Prog Neuropsychopharmacol Biol Psychiatry 1987;11:121-127.

25. Perdersen CA, Ascher JA, Monroe YL, Prange AJ Jr. Oxytocin induces maternal behavior in virgin female rats. Science 1982;216: $648-650$

26. Insel TR. Oxytocin: a neuropeptide for affiliation: evidence from behavioral, receptor autoradiographic, and comparative studies. Psychoneuroendocrinology 1992;17:3-35.

27. Champagne F, Diorio J, Sharma S, Meaney MJ. Naturally occurring variations in maternal behavior in the rat are associated differences in estrogen-inducible central oxytocin receptors. Proc Natl Acad Sci U S A 2001;98:12736-12741.

28. Takayanagi Y, Yoshida M, Bielsky IF, et al. Pervasive social deficits, but normal parturition, in oxytocin receptor-deficient mice. Proc Natl Acad Sci U S A 2005;102:16096-16101.

29. Young LJ, Winslow JT, Wang Z, et al. Gene targeting approaches to neuroendocrinology: oxytocin, maternal behavior, and affiliation. Horm Behav 1997;31:221-331.

30. Liu HX, Lopatina O, Higashida C, et al. Locomotor activity, ultrasonic vocalization and oxytocin level in infant CD38 knockout mice. Neurosci Lett 2008;448:67-70.

31. Choleris E, Gustafsson JA, Korach KS, Muglia LJ, Pfaff DW, Ogawa S. An estrogen-dependent four-gene micronet regulating social recognition: a study with oxytocin and estrogen receptoralpha and -beta knockout mice. Proc Natl Acad Sci U S A 2003; 100:6192-6197.

32. Ferguson JN, Aldag JM, Insel TR, Young LJ. Oxytocin in the medial amygdala is essential for social recognition in the mouse. J Neurosci 2001;21:8278-8285.

33. Lim MM, Wang Z, Olazábal DE, Ren X, Terwilliger EF, Young LJ. Enhanced partner preference in promiscuous species by manipulating the expression of a single gene. Nature 2004;429:754757.

34. Olazábal DE, Young LJ. Variability in "spontaneous" maternal behavior is associated with anxiety-like behavior and affiliation in naïve juvenile and adult female prairie voles (Microtus ochrogaster). Dev Psychobiol 2005;47:166-178.

35. Young KA, Liu Y, Wang Z. The neurobiology of social attachment: a comparative approach to behavioral, neuroanatomical, and neurochemical studies. Comp Biochem Physiol C Toxicol Pharmacol 2008; 148:401-410.

36. Getz LL, Hofmann JR. Social organization in free living prairie voles, Microtus ochrogaster. Behav Ecol Sociobiol 1986;18:275282.

37. Getz LL, Carter CS, Gavish L. The mating system of the prairie vole Microtus ochrogaster: field and laboratory evidence for pairbonding. Behav Ecol Sociobiol 1981;8:189-194.

38. Pizzuto T, Getz LL. Female prairie voles (Microtus ochrogaster) fail to form a new pair after loss of mate. Behav Proc 1998;43: $79-96$.

39. Getz LL, Carter CS. Prairie-vole partnerships. Am Sci 1996;84: $56-62$.
40. Insel TR, Shapiro LE. Oxytocin receptor distribution reflects social organization in monogamous and polygamous voles. Proc Natl Acad Sci U S A 1992;89:5981-5985.

41. Williams JR, Insel TR, Harbaugh CR, Carter CS. Oxytocin administered centrally facilitates formation of a partner preference in female prairie voles (Microtus ochrogaster). J Neuroendocrinol 1994;6:247-250.

42. Insel TR, Hulihan TJ. A gender-specific mechanism for pair bonding: oxytocin and prater preference formation in monogamous voles. Behav Neurosci 1995;109:782-789.

43. Olazábal DE, Young LJ. Oxytocin receptors in the nucleus accumbens facilitated "spontaneous" maternal behavior in adult female prairie voles. Neuroscience 2006;141:559-568.

44. Meisenberg G, Simmons WH. Centrally mediated effects of neurohypophyseal hormones. Neurosci Biobehav 1983;7:263-280.

45. Drago F, Pedersen CA, Caldwell JD, Prange AJ Jr. Oxytocin potently enhances novelty-induced grooming behavior in the rat. Brain Res 1986;368:287-295.

46. Insel TR, Winslow JT. Central administration of oxytocin modulates the infant rat's response to social isolation. Eur J Pharmacol 1991;203:149-152.

47. Nelson E, Alberts JR. Oxytocin-induced paw sucking in infant rats. Ann N Y Acad Sci 1997;807:543-545.

48. Van Wimersma Greidanus TB, Kroodsma JM, Pot ML, Stevens M, Maigret C. Neurohypophyseal hormones and excessive grooming behaviour. Eur J Pharmacol 1990;187:1-8.

49. McDougle CJ, Barr LC, Goodman WK, Price LH. Possible role of neuropeptides in obsessive compulsive disorder. Psychoneuroendocrinology 1999;24:1-24.

50. Crawley JN. Designing mouse behavioral tasks relevant to autisticlike behaviors. Ment Retard Dev Disabil Res Rev 2004;10:248258.

51. Ashwood P, Wills S, Van de Water J. The immune response in autism: a new frontier for autism research. J Leukoc Biol 2006; $80: 1-15$.

52. Kosfeld M, Heinrichs M, Zak PJ, Fischbacher U, Fehr E. Oxytocin increases trust in humans. Nature 2005;435:673-676.

53. Baumgartner T, Heinrichs M, Vonlanthen A, Fischbacher U, Fehr E. Oxytocin shapes the neural circuitry of trust and trust adaptation in humans. Neuron 2008;58:639-650.

54. Domes G, Heinrichs M, Michel A, Berger C, Herpertz SC. Oxytocin improves "mind-reading" in humans. Biol Psychiatry 2007; 61:731-733.

55. Baron-Cohen S, Wheelwright S, Hill J, Raste Y, Plumb I. The "Reading the Mind in The Eyes" Test revised version: a study with normal adults, and adults with Asperger syndrome or high-functioning autism. J Child Psychol Psychiatry 2001;42:241-251.

56. Theodoridou A, Rowe AC, Penton-Voak IS, et al. Oxytocin and social perception: oxytocin increases perceived facial trustworthiness and attractiveness. Horm Behav 2009;56:128-132.

57. Heinrichs M, Baumgartner T, Kirschbaum C, Ehlert U. Social support and oxytocin interact to suppress cortisol and subjective responses to psychosocial stress. Biol Psychiatry 2003;54:13891398.

58. Modahl C, Green L, Fein D, et al. Plasma oxytocin levels in autistic children. Biol Psychiatry 1998;43:270-277.

59. Jansen LM, Gispen-De Wied CC, Wiegant VM, Westenberg HG, Lahuis BE, van Engeland H. Autonomic and neuroendocrine responses to a psychosocial stressor in adults with autistic spectrum disorder. J Autism Dev Disord 2006;36:891-899.

60. Green L, Fein D, Modahl C, Feinstein C, Waterhouse L, Morris M. Oxytocin and autistic disorder: alterations in peptide forms. Biol Psychiatry 2001;50:609-613.

61. Bartz J, Young L. Oxytocin, social cognition, and autism. 2010 (in press).

62. Taylor SE, Gonzaga GC, Klein LC, Hu P, Greendale GA, Seeman TE. Relation of oxytocin to psychological stress responses and hypothalamic-pituitary-adrenocortical axis activity in older women. Psychosom Med 2006;68:238-245.

63. Turner RA, Altemus M, Enos T, Cooper B, McGuinness T. Preliminary research on plasma oxytocin in normal cycling women: investigating emotion and interpersonal distress. Psychiatry 1999; 62:97-113. 
64. Hoge EA, Pollack MH, Kaufman RE, Zak PJ, Simon NM. Oxytocin levels in social anxiety disorder. CNS Neurosci Ther 2008; 14:165-170.

65. Kirsch P, Esslinger C, Chen Q, et al. Oxytocin modulates neural circuitry for social cognition and fear in humans. J Neurosci 2005; 25:11489-11493.

66. Domes G, Heinrichs M, Gläscher J, Büchel C, Braus DF, Herpertz SC. Oxytocin attenuates amygdala responses to emotional faces regardless of valence. Biol Psychiatry 2007;62:1187-1190.

67. Petrovic P, Kalisch R, Singer T, Dolan RJ. Oxytocin attenuates affective evaluations of conditioned faces and amygdala activity. J Neurosci 2008;28:6607-6615.

68. Singer T, Snozzi R, Bird G, et al. Effects of oxytocin and prosocial behavior on brain responses to direct and vicariously experienced pain. Emotion 2008;8:781-791.

69. Domes G, Lischke A, Berger C, et al. Effects of intranasal oxytocin on emotional face processing in women. Psychoneuroendocrinology 2010;35:83-93.

70. Lintas C, Persico AM. Autistic phenotypes and genetic testing: state-of-the-art for the clinical geneticist. J Med Genet 2009;46: $1-8$.

71. Folstein S. Twin and adoption studies in child and adolescent psychiatric disorders. Curr Opin Pediatr 1996;8:339-347.

72. Bailey A, Le Couteur A, Gottesman I, et al. Autism as a strongly genetic disorder: evidence from a British twin study. Psychol Med 1995;25:63-77.

73. Wu S, Jia M, Ruan Y, et al. Positive association of the oxytocin receptor gene $(O X T R)$ with autism in the Chinese Han population. Biol Psychiatry 2005;58:74-77.

74. Jacob S, Brune CW, Carter CS, Leventhal BL, Lord C, Cook EH Jr. Association of the oxytocin receptor gene $($ OXTR $)$ in Caucasian children and adolescents with autism. Neurosci Lett 2007;417:6-9.
75. Lerer E, Levi S, Salomon S, Darvasi A, Yirmiya N, Ebstein RP. Association between the oxytocin receptor (OXTR) gene and autism: relationship to Vineland Adaptive Behavior Scales and cognition. Mol Psychiatry 2008;13:980-988.

76. Wermter AK, Kamp-Becker I, Hesse P, et al. Evidence for the involvement of genetic variation in the oxytocin receptor gene $($ OXTR $)$ in the etiology of autistic disorders on high-functioning level. Am J Med Genet B Neuropsychiatr Genet 2010; 153B:629-639.

77. Gregory SG, Connelly JJ, Towers A, et al. Genomic and epigenetic evidence for oxytocin receptor deficiency in autism. BMC Med 2009;7:62.

78. Hobson RP, Ouston J, Lee A. Emotion recognition in autism: coordinating faces and voices. Psychol Med 1988;18:911-923.

79. Rutherford MD, Baron-Cohen S, Wheelwright S. Reading the mind in the voice: a study with normal adults and adults with Asperger syndrome and high functioning autism. J Autism Dev Disord 2002;32:189-194.

80. Guastella AJ, Einfeld SL, Gray KM, et al. Intranasal oxytocin improves emotion recognition for youth with autism spectrum disorders. Biol Psychiatry 2010;67:692-694.

81. Neumann D, Spezio ML, Piven J, Adolphs R. Looking you in the mouth: abnormal gaze in autism resulting from impaired top-down modulation of visual attention. Soc Cogn Affect Neurosci 2006;1: 194-202.

82. Guastella AJ, Mitchell PB, Dadds MR. Oxytocin increases gaze to the eye region of human faces. Biol Psychiatry 2007;1:3-5.

83. Rimmele U, Hediger K, Heinrichs M, Klaver P. Oxytocin makes a face in memory familiar. J Neurosci 2009;29:38-42.

84. Benvenuto A, Moavero R, Alessandrelli R, Manzi B, Curatolo P. Syndromic autism: causes and pathogenetic pathways. World J Pediatr 2009;5:169-176. 